\title{
Metabonomics Study on the Effect of Traditional Chinese Medicines Feed Addition on Growth Performance and Serum Metabolic Profile of Juvenile Chinese Softshell Turtle (Pelodiscus Sinensis Wiegmann) using UPLC-Triple /TOF-MS Analysis
}

Mingsong Xiao ( $\nabla$ xiaomingsong2004@126.com )

Anhui Science and Technology University https://orcid.org/0000-0002-0168-8834

Fangyin Bao

Anhui Science and Technology University

Yuliang Wang

Anhui Science and Technology University

Huabin Liu

Anhui Science and Technology University

Jixiang He

Anhui Academy of Agricultural Sciences

Research

Keywords: Pelodiscus sinensis, UPLC-Triple-TOF-MS/MS, Metabolomics analysis, TCMs, MetPA

Posted Date: July 22nd, 2020

DOI: https://doi.org/10.21203/rs.3.rs-40833/v2

License: (c) (i) This work is licensed under a Creative Commons Attribution 4.0 International License.

Read Full License 
The authors have withdrawn this preprint from Research Square 